\title{
Carbohydrate catabolic flexibility in the mammalian intestinal commensal Lactobacillus ruminis revealed by fermentation studies aligned to genome annotations
}

\author{
Michelle M O' Donnell ${ }^{1,2}$, Brian M Forde ${ }^{2}$, B Neville ${ }^{2}$, Paul R Ross' ${ }^{1}$ Paul W O' Toole ${ }^{2^{*}}$
}

From 10th Symposium on Lactic Acid Bacterium

Egmond aan Zee, the Netherlands. 28 August - 1 September 2011

\begin{abstract}
Background: Lactobacillus ruminis is a poorly characterized member of the Lactobacillus salivarius clade that is part of the intestinal microbiota of pigs, humans and other mammals. Its variable abundance in human and animals may be linked to historical changes over time and geographical differences in dietary intake of complex carbohydrates.

Results: In this study, we investigated the ability of nine L. ruminis strains of human and bovine origin to utilize fifty carbohydrates including simple sugars, oligosaccharides, and prebiotic polysaccharides. The growth patterns were compared with metabolic pathways predicted by annotation of a high quality draft genome sequence of ATCC 25644 (human isolate) and the complete genome of ATCC 27782 (bovine isolate). All of the strains tested utilized prebiotics including fructooligosaccharides (FOS), soybean-oligosaccharides (SOS) and 1,3:1,4- $\beta$-D-glucooligosaccharides to varying degrees. Six strains isolated from humans utilized FOS-enriched inulin, as well as FOS. In contrast, three strains isolated from cows grew poorly in FOS-supplemented medium. In general, carbohydrate utilisation patterns were strain-dependent and also varied depending on the degree of polymerisation or complexity of structure. Six putative operons were identified in the genome of the human isolate ATCC 25644 for the transport and utilisation of the prebiotics FOS, galacto-oligosaccharides (GOS), SOS, and 1,3:1,4- $\beta$-D-Gluco-oligosaccharides. One of these comprised a novel FOS utilisation operon with predicted capacity to degrade chicory-derived FOS. However, only three of these operons were identified in the ATCC 27782 genome that might account for the utilisation of only SOS and 1,3:1,4- $\beta$-D-Gluco-oligosaccharides.
\end{abstract}

Conclusions: This study has provided definitive genome-based evidence to support the fermentation patterns of nine strains of Lactobacillus ruminis, and has linked it to gene distribution patterns in strains from different sources.

Furthermore, the study has identified prebiotic carbohydrates with the potential to promote L. ruminis growth in vivo.

\section{Background}

Immediately following birth, humans are colonised by a variety of bacteria which form the gastrointestinal tract microbiota [1]. Lactic Acid bacteria (LAB), which include Lactobacillus spp., are a subdominant element of the microbiota of humans and animals [2].

\footnotetext{
* Correspondence: pwotoole@ucc.ie

2Department Microbiology, University College Cork, Ireland

Full list of author information is available at the end of the article
}

Lactobacillus ruminis is a LAB which is part of the autochthonous microbiota in the intestines of both humans [3], and pigs [4] and it has also been isolated from the bovine rumen [5]. L. ruminis is a low $\mathrm{G}+\mathrm{C}$ Gram positive bacillus [6]. It is a candidate probiotic organism (see below), since it has been reported to have immunomodulatory characteristics [7], specifically the ability to induce Nuclear Factor Kappa B (NF- $\kappa$ B) in the absence of lipopolysaccharide production and to activate Tumour Necrosis Factor alpha (TNF $\alpha)$ production in 
THP-1 monocytes [7]. Unusually, some strains of $L$. ruminis are motile [5]. Limited studies have identified some of the carbohydrates utilised by L. ruminis which include cellobiose and raffinose $[5,6,8]$. However, little information is available about the fermentation of oligosaccharides/prebiotics by Lactobacillus ruminis.

There is growing interest in modulating the human microbiota using dietary supplements including probiotics and prebiotics. Probiotics are defined as "live microorganisms which when administered in adequate amounts confer a health benefit on the host" [9]. However, maintained ingestion of probiotic cultures is generally required to sustain the probiotic effect, with only some of the inoculum surviving gastrointestinal transit, and the vast majority of surviving bacteria shed days after ingestion [10]. For this reason there has been an increasing research effort expended in the area of prebiotics in order to extend the persistence of particular bacteria (mainly bifidobacteria) in the intestine. Prebiotics are "selectively fermented ingredients that result in specific changes in the composition and/or activity of the gastrointestinal microbiota, thus conferring benefit (s) upon host health" [11]. To be considered a prebiotic, the compound has to resist hydrolysis by gastrointestinal tract enzymes and pass into the large intestine, where ideally it promotes the growth of commensal bacteria [12]. The fermentation of prebiotics in the colon is largely influenced by the type of sugar monomer, the degree of polymerisation and the nature of the glycosidic bonds between the sugar moieties [13]. The constituent sugars of the majority of prebiotics are monosaccharides such as glucose, fructose, galactose and xylose [14]. The degree of polymerisation (DP) of prebiotics can vary from as low as two for lactulose and in excess of 23 for chicory-derived inulin [15]. Humans lack the gastrointestinal enzymes necessary to degrade many of the glycosidic bonds between the sugar units of compounds that are prebiotics, which accounts for their resistance to hydrolysis [14]. A number of enzymes produced by colonic commensal bacteria may hydrolyse these bonds. These glycosyl hydrolase (GH) enzymes include $\beta$-Glucosidases, $\alpha$-Glucosidases, $\beta$-Fructofuranosidases, $\beta$-Galactosidases and $\alpha$-Galactosidases [16-18].

Studies of other Lactobacillus species have identified a variety of genetic systems that encode the ability to utilize carbohydrates of varying complexity. $\beta$-fructofuranosidase is responsible for the hydrolysis of FOS, and this activity was identified in L. plantarum WCFS1 [19], $L$. acidophilus NCFM [20], and L. paracasei 1195 [21]. $\beta$ galactosidases involved in lactose degradation were characterised in L. sakei[22], L. bulgaricus[23], L. coryniformis[24] and $L$. reuteri[25]. $\beta$-glucosidase activity (which is responsible for the hydrolysis of 1,4- $\beta$-D-Glucans like cellobiose) has been identified in L. plantarum[26]. $\alpha$ - galactosidases, which hydrolyse $\alpha$-galactosides like raffinose, stachyose and melibiose, were identified in $L$. plantarum ATCC 8014 [27] and L. reuteri[28]. Moreover, several $\alpha$-glucosidases have been characterised in L. brevis[29], L. acidophilus[30] and L. pentosus[31].

In this study, we describe the fermentation profiles of nine strains of Lactobacillus ruminis. The interpretation of the carbohydrate utilisation profiles generated was complemented by the annotation of carbohydrate utilisation genes in the genomes of L. ruminis ATCC 25644 and ATCC 27782.

\section{Results}

\section{Growth of $L$. ruminis in media containing diverse carbon sources}

A carbohydrate utilisation profile for each of nine strains of L. ruminis on fifty carbohydrates was established as described in Methods. Additional file 1 summarizes the data, with individual strain data in Additional Files 2, 3, 4, 5, 6, 7, 8, 9, 10. In summary, there was significant variation with respect to carbohydrate fermentation profiles at the strain level. Moderate growth was observed for strains L5 and S21 when grown on $\alpha$-galactosides (melibiose, raffinose, stachyose) and $\beta$-glucosides ( $\beta$-glucotriose $B$, cellobiose) (Additional file 1$)$. The majority of bovine isolates could poorly utilize fructooligosaccharides, except for ATCC 27781 with Beneo P95 and Raftilose P95. Moderate growth was observed for the majority of isolates with galactooligosaccharides (GOS, GOS-inulin, lactose, lactulose). All strains were able to ferment $\beta$-Glucotriose B, cellobiose, galactose, glucose, maltose, mannose, melibiose, raffinose, stachyose and sucrose (Additional file $1)$. Some strains showed a distinctly higher ability to utilize specific carbohydrates e.g. fructose by strains L5 and S21, (Additional Files 2 and 3); lactose by strains S23, ATCC 25644 and ATCC 27780T (Additional Files 4, 7 and 8); raffinose by ATCC 27781 (Additional File 8); and Raftilose P95 by strain S36 (Additional File 5).

\section{Growth and fermentation analysis of human and bovine- derived $L$. ruminis type strains}

Table 1 shows the final cell numbers and culture-medium $\mathrm{pH}$ values reached for the two strains ATCC 25644 (human isolate) and ATCC 27782 (bovine isolate), in the presence of various carbohydrates and prebiotics for 24 h. L. ruminis ATCC 25644 reached the highest cell density $\left(8.9 \times 10^{8} \mathrm{cfu} / \mathrm{ml}\right)$ when grown on Raftilose Synergy 1 which coincided with the lowest culture medium $\mathrm{pH}$ value of 4.86 . ATCC 27782 reached the highest cell density values $\left(2.7 \times 10^{8} \mathrm{cfu} / \mathrm{ml}\right)$ when grown on Beta Glucotriose B, and fermentation resulted in a culture medium $\mathrm{pH}$ value of 5.19 following 24 hours incubation. This was far higher than cellobiose, the other beta- 
Table 1 Growth and fermentation analysis of L. ruminis strains ATCC 25644 (human isolate) and ATCC 27782 (bovine isolate).

\begin{tabular}{|c|c|c|c|c|c|}
\hline \multirow[t]{2}{*}{ Carbohydrate type } & \multirow[t]{2}{*}{ Carbohydrate } & \multicolumn{2}{|c|}{ ATCC 25644} & \multicolumn{2}{|c|}{ ATCC 27782} \\
\hline & & $\mathrm{Cfu} / \mathrm{ml}$ & $\mathrm{pH}^{*}$ & $\mathrm{Cfu} / \mathrm{ml}$ & $\mathrm{pH}^{*}$ \\
\hline & Cellobiose & $2.40 \times 10^{8}$ & 5.21 & $7.00 \times 10^{6}$ & 5.13 \\
\hline \multirow[t]{2}{*}{ Disaccharide } & Lactulose & $3.20 \times 10^{8}$ & 4.99 & 0 & 6.53 \\
\hline & Lactose & $2.76 \times 10^{8}$ & 4.76 & 0 & 6.57 \\
\hline \multirow[t]{2}{*}{ Monosaccharide } & Glucose & $4.39 \times 10^{8}$ & 4.86 & $1.53 \times 10^{8}$ & 4.85 \\
\hline & Beta Glucotriose B & $4.05 \times 10^{8}$ & 5.17 & $2.66 \times 10^{8}$ & 5.19 \\
\hline \multirow[t]{2}{*}{ Oligosaccharide } & Raftilose Synergy 1 & $8.90 \times 10^{8}$ & 5.01 & $1.35 \times 10^{7}$ & 6.04 \\
\hline & Raftilose P95 & $2.91 \times 10^{8}$ & 5.28 & $2.51 \times 10^{6}$ & 5.42 \\
\hline Tetrasaccharide & Stachyose & $3.94 \times 10^{8}$ & 5.13 & $2.37 \times 10^{8}$ & 5.11 \\
\hline Trisaccharide & Raffinose & $3.24 \times 10^{8}$ & 5.2 & $1.40 \times 10^{8}$ & 5.2 \\
\hline
\end{tabular}

*. $\mathrm{pH}$ value of culture medium after $24 \mathrm{~h}$ growth in indicated carbon source. Values tabulated are the average of two replicates carried out on separate days.

glucoside tested, although the final $\mathrm{pH}$ of both cultures was very similar, and the medium was buffered in the same way as MRS.

\section{Annotation of carbohydrate pathways in the $L$. ruminis genome}

A high-quality draft genome sequence was generated for L. ruminis ATCC 25644 and a finished genome sequence was generated for ATCC 27782, as described in Methods. The complete functional and comparative analysis of these genomes will be described elsewhere (Forde et al., in preparation; Neville et al., in preparation). A draft sequence of ATCC 25644 has also been generated by the Human Microbiome Project [32]; however it has a different scaffold structure and assembly statistics to that which we generated for ATCC 25644, and for that reason was not used in the current study. The carbohydrate utilisation genes of ATCC 25644 and ATCC 27782 were annotated by manual curation in conjunction with KEGG Automatic Annotation Server (KAAS). L. ruminis-specific Kyoto Encyclopaedia of Genes and Genomes (KEGG) maps were generated based upon our annotated genome sequences that we analyzed with KAAS. As a representative example, the galactose metabolic pathway (for both sequenced $L$. ruminis genomes) is presented in Figure 1. It demonstrates the predicted reliance on glycosyl hydrolases to ferment carbohydrates in L. ruminis as well as highlighting the fermentable $\alpha$ and $\beta$-galactosides.

Sixteen major pathways or systems involved in carbohydrate utilization were annotated in both genomes, and are shown in Additional Files 11, 12, 13, 14, 15, 16, 17, $18,19,20,21,22,23,24,25,26$. These include those for glycolysis, pentose and glucuronate interconversions, fructose and mannose utilization, starch and sucrose. Of the sixteen pathways identified, eight are considered partial pathways (Additional Files 11, 12, 13, 14, 15, 16, $17,18,19,20,21,22,23,24,25,26)$.

\section{Identification of Glycosyl Hydrolases}

Glycosyl hydrolases are key to prebiotic utilization, and can also be manipulated to synthesize prebiotics. Twenty glycosyl hydrolases were annotated in the genome of ATCC 25644, and fourteen were annotated in the genome of ATCC 27782. The glycosyl hydrolases include $\alpha$-amylase (EC 3.2.1.1), endo-1,4- $\beta$-xylanase (EC 3.2.1.8), oligo-1,6-glucosidase (EC 3.2.1.10), lysozyme (EC 3.2.1.17), $\alpha$-glucosidase (EC 3.2.1.20), $\beta$-glucosidase (EC 3.2.1.21), $\alpha$-galactosidase (EC 3.2.1.22), $\beta$-galactosidase (EC 3.2.1.23), $\beta$-fructofuranosidase (EC 3.2.1.26), $\beta$ $\mathrm{N}$-acetylhexosaminidase (EC 3.2.1.52), glucan 1,6- $\alpha$-glucosidase (EC 3.2.1.70), 6-phospho- $\beta$-glucosidase (EC 3.2.1.86) and neopullalanase (EC 3.2.1.135). The majority of these enzymes are present in ATCC 27782 with the exceptions of $\alpha$-amylase, oligo-1,6-glucosidase and $\beta$ galactosidase.

\section{Identification of putative genes and operons involved in prebiotic utilisation}

The sequenced L. ruminis genomes were extensively scrutinized to identify putative operons involved in carbohydrate transport and utilisation. Specificity of substrate was based upon manual curation of the annotated region, including reference to BLAST identity to functionally characterized homologues, genetic neighbourhood analysis, and protein motif matching. Six putative prebiotic utilisation operons were annotated in the $L$. ruminis ATCC 25644 genome (human isolate; Figure 2), 


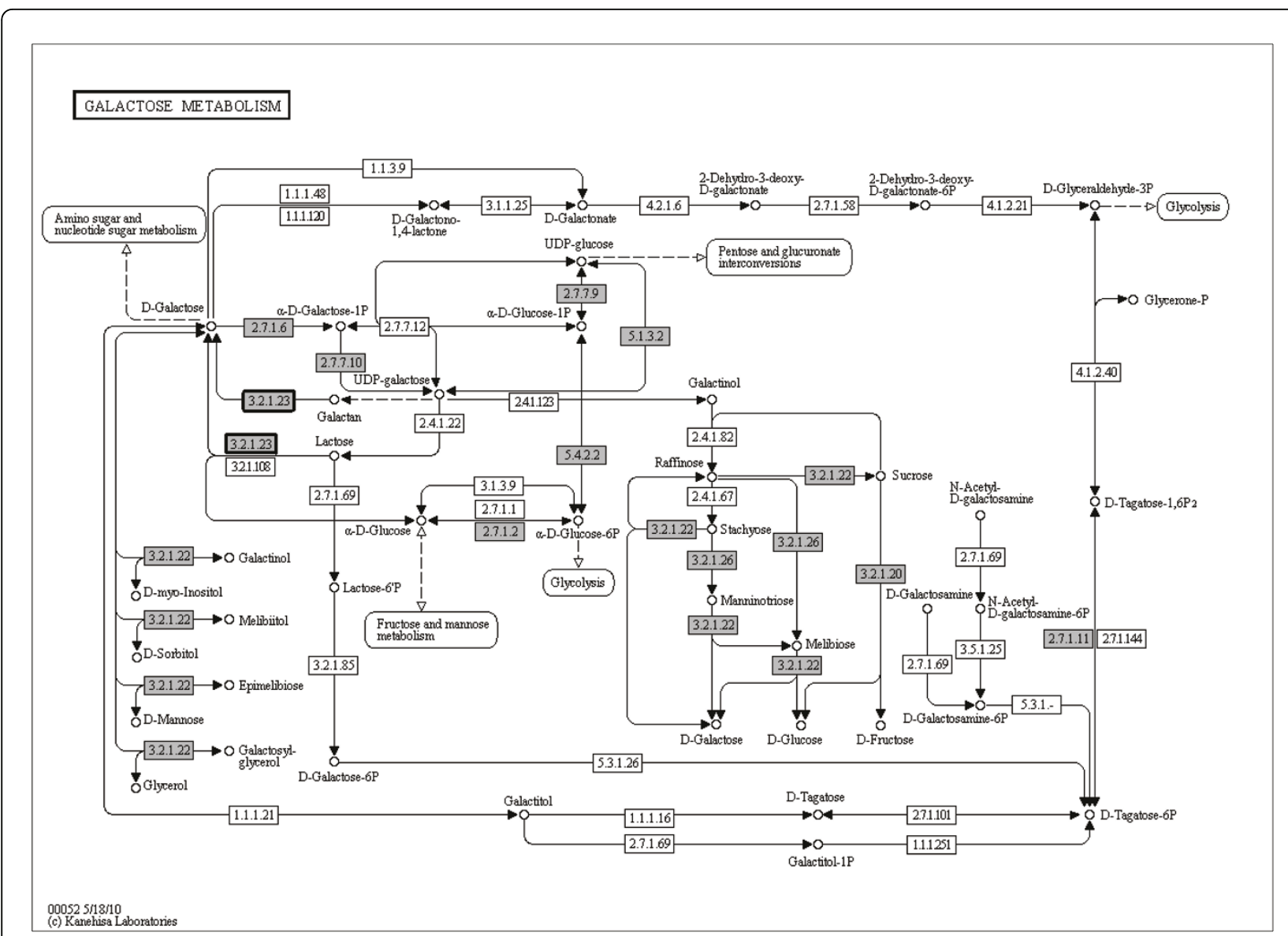

Figure 1 Galactoside utilisation metabolic map for L. ruminis ATCC $\mathbf{2 5 6 4 4}$ and ATCC $\mathbf{2 7 7 8 2}$. Grey boxes, enzymes present in both ATCC 25644 and ATCC 27782; Grey boxes with emphasised black border, enzymes present in ATCC 25644 and absent from ATCC 27782.

only three of which were identified in the bovine isolate ATCC 27782 (Additional File 27). Most of the operons are flanked by predicted rho-independent transcriptional terminators (Figure 2), and these operons constitute one to two transcriptional units, with a gene for a LacI-type transcriptional regulator in four of six cases.

We annotated a predicted FOS utilization operon only in the human isolate L. ruminis ATCC 25644. $\beta$-fructofuranosidase, a Glycosyl hydrolase (GH) family 32 enzyme [16], has been identified as the key enzyme in operons involved in FOS utilisation in other Lactobacillus species [19-21]. This activity is predicted to be encoded by the L. ruminisbfrA gene, which is linked to a presumptive oligosaccharide symporter gene. The ATCC 25644 genome was also distinguished by having two additional operons for lactose/galactose utilization (Figure 2). The genomes of both strains harboured operons predicted to confer utilization of sucrose, cellobiose and raffinose. As well as the $\beta$-fructofuranosidase $(s a c A)$ in the sucrose operon, genes for an amylopullalanase $(a m y B)$ and an $\alpha$-glucosidase (malZ) are also contiguous and are potentially co-transcribed with the sucrose operon, but do not have a predicted function in the hydrolysis of sucrose or FOS (Figure 2B).

The cellobiose operon is predicted to be responsible for the transport and hydrolysis of both cellobiose and 1,3:1,4$\beta$-D-Glucan hydrolysates, and in L. ruminis it appears to involve two $\beta$ glucosidases (Figure 2) that belong to the GH1 family of glycosyl hydrolases [16]. The amino acid sequence of $\mathrm{BglB}$ and $\mathrm{BglB}_{2}$ showed $70 \%$ and $77 \%$ identity to the $\beta$-glucosidases identified in the genomes of $L$. helveticus DPC 4571 and L. ultunensis DSM 16047, respectively. The products of the raffinose operon (Fig. 2D; also present in ATCC 27782) are predicted to have the additional ability to breakdown melibiose and stachyose. All of the glycolytic enzymes discussed above lack predicted transmembrane domains (TMD) and therefore most likely require import of their respective substrates.

\section{Predicted carbohydrate transporters}

A relationship exists between the genomic association of genes and the functional interaction of the proteins they 
A

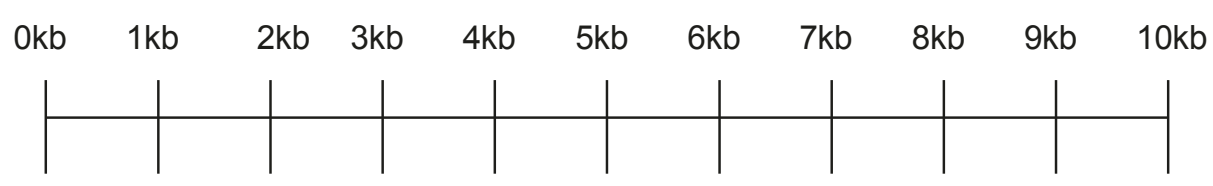

B

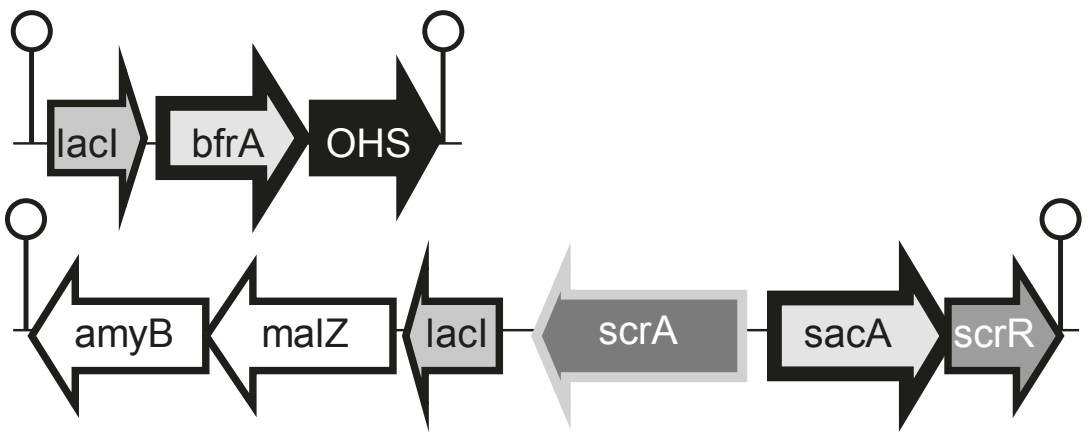

C

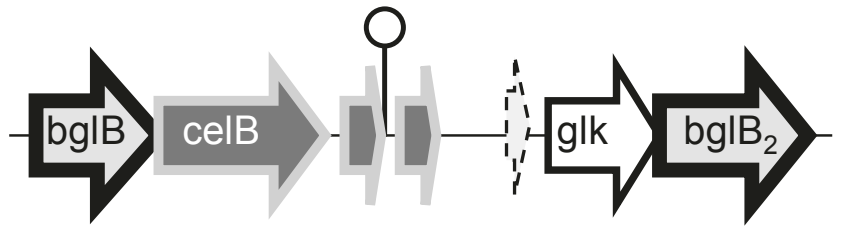

celA celC

D

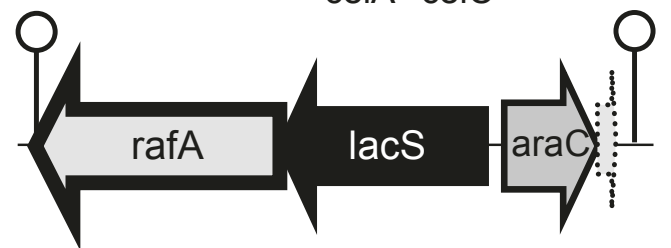

E

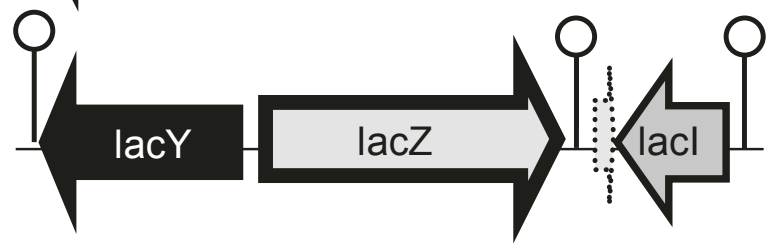

$\mathbf{F}$

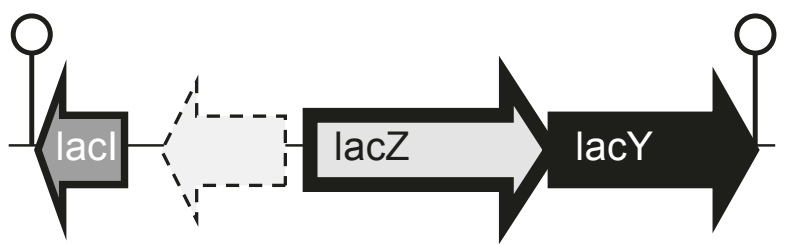

Figure 2 Putative operons for the predicted utilisation of carbohydrates in L. ruminis ATCC 25644. Predicted substrates are A, FOS; B, Sucrose; C, Cellobiose; D, raffinose; E, lactose/galactose; F, lactose/galactose operon. Light grey arrows with thick black border, glycosyl hydrolase family enzyme; Black arrows, major facilitator superfamily transporters; Medium grey arrows, transcriptional regulators; Dark grey arrows with thick grey border, phosphotransferase system transporters; Lollipops, rho-dependent transcriptional terminators; White arrows with dashed surround, transposases; white arrows with dotted surround, hypothetical proteins; White arrows with black continuous surround, potentially co-transcribed enzymes. Operons B, C and D were also annotated in the ATCC 27782 genome (see Additional File 27: Figure 1).

encode [33]. To refine our annotation of the carbohydrate utilisation operons, we therefore performed a detailed analysis of the predicted transporter proteins encoded by the contiguous genes. As for hydrolases, specificity of substrate was predicted based upon an integrated analysis of the annotated region, including reference to BLAST identity to functionally characterized homologues, linked genes, and protein motif matching. Putative carbohydrate transporters were analysed with transmembrane prediction software, with 14 and 10 transporters identified in the genome sequences of L. ruminis ATCC 25644 and 27782, respectively (Table 2 ). The predicted carbohydrate 
Table 2 Transmembrane domains (TMD) of the predicted carbohydrate transport proteins in Lactobacillus ruminis

\begin{tabular}{|c|c|c|c|c|c|c|}
\hline \multirow[t]{2}{*}{ Family } & \multirow[t]{2}{*}{ Gene } & \multicolumn{2}{|c|}{ Locus number $^{\mathrm{a}}$} & \multirow[t]{2}{*}{ Predicted substrate } & \multicolumn{2}{|l|}{$\mathrm{TMD}^{\mathrm{b}}$} \\
\hline & & ATCC 25644 & ATCC 27782 & & ATCC 25644 & ATCC 27782 \\
\hline $\mathrm{OHS}$ & lacY & ANHS_218 & - & FOS & 12 & - \\
\hline \multirow[t]{3}{*}{$\mathrm{GPH}$} & lacY & $\begin{array}{l}\text { ANHS_744c } \\
\text { ANHS_924 }\end{array}$ & - & Lactose, galactose, galactan & 12 & - \\
\hline & lacs & ANHS_783 & LRC_18250 & Raffinose, stachyose, melibiose & 12 & 12 \\
\hline & ugpE & ANHS_648 & LRC_16940 & Glycerol & 6 & 6 \\
\hline \multirow[t]{5}{*}{$A B C$} & ugpA & ANHS_649C & LRC_16950 & Glycerol & 6 & 6 \\
\hline & malG & ANHS_839C & LRC_18720 & Maltose & 6 & 6 \\
\hline & malF & ANHS_840C & LRC_18730 & Maltose & 8 & 8 \\
\hline & $\operatorname{manY}$ & ANHS_242 & LRC_18860 & Mannose & 7 & 7 \\
\hline & $\operatorname{manZ}$ & ANHS_243 & LRC_18850 & Mannose & 5 & 4 \\
\hline \multirow[t]{4}{*}{ PTS } & $s c r A$ & ANHS_846C & LRC_18780 & Sucrose, FOS & 8 & 8 \\
\hline & fruA & ANHS_1075 & LRC_00800 & Fructose & 9 & 9 \\
\hline & celB & ANHS_1218 & LRC_02240 & Cellobiose & 10 & 10 \\
\hline & gluA & ANHS_851C & LRC_18820 & Glucose & 9 & 9 \\
\hline
\end{tabular}

a. Locus number in draft genome sequences

b. TMD: predicted trans-membrane domains, as described in Materials \& Methods

transporters belong to the ATP-binding Cassette family $(\mathrm{ABC})$, the Glycoside-Pentoside-Hexuronide cation symporter family (GPH), the Oligosaccharide $\mathrm{H}^{+}$Symporter (OHS) and the Phosphotransferase System (PTS). Transmembrane domain (TMD) numbers are generally indicative of the type of carbohydrate transporter, with some exceptions [34]. ABC transporters have on average 10-12 TMD but this can be highly variable. PTS transporters have been identified with up to 10 TMD (this study). GPH and OHS transporters (both being Major Facilitator Superfamily transporters) generally have 12 TMD [34]. In ATCC 25644, three GPH transporters were identified (Table 2) and these are predicted to transport the $\beta$-galactosides (lactose, galactose, lactulose and GOS) and the $\alpha$ galactosides (raffinose, melibiose and stachyose). However, in ATCC 27782 only one GPH transporter was identified, which was predicted to transport $\alpha$-galactosides. The OHS identified in the genome of ATCC 25644 is adjacent to a $\beta$-fructofuranosidase and may be involved in transporting FOS. Both genomes encode six predicted PTS transporters, which potentially transport mannose, sucrose, fructose, cellobiose and glucose. In both L. ruminis genome sequences, four $\mathrm{ABC}$ transporters were identified, with the putative substrates identified as mannose and glycerol-3phosphate. All of the transporters identified in each genome had associated metabolic genes located either upstream or downstream in the genome, and the majority were arranged in operons. Both genomes also encoded proteins for glucose uptake (with TMD counts of 5 and 9 in ATCC 25644 and ATCC 27782, respectively), and a simple sugar transport system permease protein which was predicted to transport monosaccharides like galactose.

\section{Discussion}

We consider L. ruminis as a candidate probiotic, which we are also investigating as a potential responder for prebiotic/symbiotic supplementation in humans and animals. Several studies have identified L. ruminis in the gastrointestinal tract of humans [35-37]. L. ruminis was isolated from the bovine rumen [5], from the pig [4,8]], chickens [38], sheep [39], Svalbard reindeer [40], horses [41-43], cats [44,45], dogs [46] and parrots [47]. L. ruminis thus appears to be variably present in the microbiota of humans and many domesticated animals.

L. ruminis was previously described as a homofermentative bacterium, with the ability to ferment amygdalin, cellobiose, galactose, maltose, mannose, melibiose, raffinose, salicin, sorbitol and sucrose [48]. In the current study, the nine strains of $L$. ruminis were unable to utilise sorbitol as a carbon source. L. ruminis has also been reported to have the ability to ferment D-ribose [49]. However, we observed no growth for any of the nine $L$. ruminis strains when cultured in cfMRS supplemented with ribose. ATCC 27782 lacks a transaldolase gene (and the draft genome sequence suggests ATCC 25644 also lacks this gene), which would account for inability to utilise any of the pentose sugars tested. All of the $L$. ruminis strains tested (with the exception of ATCC 27782 which lacks a lac $Z$ gene) had strong growth in lactose. This contrasts with a previous study, where 
moderate growth was recorded on lactose [48]. It has also been reported that L. ruminis showed a strain dependent fermentation of starch [50], and very little growth was recorded for any of the strains tested here.

As a species, L. ruminis is generally able to ferment prebiotic compounds including FOS, GOS, lactulose, 1,3:1,4 $\beta$-D-Glucooligosaccharides, raffinose and stachyose. Only one strain, S36 was capable of (weakly) fermenting the prebiotic disaccharide palatinose. Palatinose is made by enzymatic rearrangement of the glycosidic linkages present in sucrose from an $\alpha-1,2$-fructoside to an $\alpha-1,6$-fructoside [51]. This suggests that the catalytic enzymes involved in sucrose utilisation may no longer be able to degrade the $\alpha-1,6$-fructoside linkage in this disaccharide. The majority of $L$. ruminis strains achieved higher cell densities when grown on the prebiotic carbohydrates raffinose, lactulose, FOS, GOS and stachyose than when grown in other mono- and disaccharide carbohydrates tested. This growth pattern may be attributed to a niche for L. ruminis in the lower gastrointestinal tract (GIT). Mono and disaccharides are often unable to resist the hydrolytic action of the upper GIT, unlike prebiotics, and would not therefore be as freely available as carbon sources for L. ruminis in the large intestine. Lactulose, a disaccharide derivative of lactose, has previously been shown to support high level growth of other lactobacilli namely $L$. rhamnosus, $L$. paracasei and L. salivarius[52]. Lactulose also supported a high level of growth for the majority of L. ruminis strains. The $\beta$-galactosides lactulose and GOS are predicted to be transported and hydrolysed in ATCC 25644 by LacY and LacZ as part of the lactose operon. Two operons for $\beta$-galactoside utilisation were identified in the genome of ATCC 25644; however neither of these operons or any potential genetic determinants could be identified for lactose utilisation in ATCC 27782. The absence of a lactose operon in the genome may suggest an ecological niche adaptation by ATCC 27782 to an environment devoid of milk sugars.

$\beta$-glucooligosaccharides such as cellobiose are generally transported and hydrolysed using the cellobiose PTS and $\beta$-glucosidase enzymes. Both cellobiose and $\beta$-glucotriose $B$ are 1,4- $\beta$-D-glucooligosaccharides with a similar structure which allows the transport and utilisation of these carbohydrates by the products of the cellobiose operon. The bovine L. ruminis isolates, ATCC $27780 \mathrm{~T}, 27781$ and 27782 were previously reported to utilise $\beta$-glucan hydrolysates as a carbohydrate source [53], and in that study, all bovine isolates utilised $\beta$-glucan hydrolysates of DP3, and only ATCC 27780 T was unable to utilise DP4 oligosaccharide. ATCC 27781 was distinguished by being able to utilise the highest percentage of both DP3 and DP4 $\beta$ glucan. We have shown that all the strains tested in this study were able to utilise the DP3 $\beta$-glucan hydrolysates to a moderate degree. The bovine isolate ATCC $27780 \mathrm{~T}$ achieved the highest growth (data not shown) when utilizing $\beta$ glucan hydrolysate, in contrast to a previous study which identified ATCC 27781 as having the highest percentage utilisation of $\beta$-glucan oligosaccharide [53].

In previous analysis of sixteen Lactobacillus species, only L. acidophilus L3, L. acidophilus 74-2 and L. casei CRL431 were able to utilise Raftilose P95, an oligofructose [54]. In the current study, eight strains of L. ruminis were capable of utilizing Raftilose P95. In addition, L. ruminis was capable of moderate to strong fermentation of Raftilose Synergy 1, an oligofructose-enriched inulin. L. paracasei subsp. paracasei 8700:2 was previously shown to be the only strain, out of ten strains tested, that was capable of strong growth on Raftilose Synergy 1, while three other species were capable of moderate growth [55]. Based on these comparisons, $L$. ruminis may have a growth advantage over other lactobacilli in the presence of fructooligosaccharides.

A novel $\beta$-fructofuranosidase was identified in the genome of L. ruminis ATCC 25644 that potentially hydrolyses the linkages present in chicory derived fructooligosaccharides. The cognate transporter OHS was identified only in the strains isolated from humans. Transport of FOS may be transported using the sucrose PTS transporter in the bovine strains ATCC 27780 and 27781. The human isolates of $L$. ruminis apparently use an OHS to transport FOS into the cell. Both sequenced strains likely use the $\mathrm{ABC}$ transport system to transport simple carbohydrates like maltose and glycerol. The most populated class of transporter identified was the phosphotransferase system transporter, with six such systems present. However, in L. ruminis many of the fermentable carbohydrates including $\alpha$-galactosides and $\beta$-galactosides are predicted to be transported by GPH symporters. GPH transporters contain a C-terminal hydrophilic domain which interacts with the PTS system [34], which may thus be an important regulatory mechanism in L. ruminis.

\section{Conclusions}

Lactobacillus ruminis is a saccharolytic member of the intestinal microbiota capable of degrading a variety of prebiotics. Genes and operons were identified in the genomes of two sequenced strains for the hydrolysis and transport of the utilisable prebiotics. This work is the first step in the characterisation of carbohydrate metabolism, transportation and regulation in L. ruminis. Further studies will focus on the functional characterisation of the putative operons identified in this study and also in vivo studies with dietary supplementation by selected carbohydrates. Characterisation of the novel FOS degrading enzyme BfrA may facilitate applications 
including reverse engineering of the FOS degradation pathway to allow the biosynthesis of a potentially novel fructooligosaccharide.

\section{Methods}

\section{Bacterial strains and culture conditions}

Nine Lactobacillus ruminis strains were used in this study, and were obtained courtesy of Prof. Gerald Tannock, University of Otago, New Zealand. Four of these are American Type Culture Collection strains: ATCC 25644 (human isolate), ATCC 27780T, ATCC 27781 and ATCC 27782 (bovine isolates). Five human-derived L. ruminis strains, L5, S21, S23, S36 and S38 were also studied. All strains were stored at $-80^{\circ} \mathrm{C}$ in de ManRogosa-Sharpe (MRS) broth (Difco, BD, Ireland), supplemented with $25 \%(\mathrm{vol} / \mathrm{vol})$ glycerol as a cryoprotectant. Lactobacillus strains were grown anaerobically on MRS agar plates at $37^{\circ} \mathrm{C}$ for two days. Growth tests were initiated by growing Lactobacillus strains anaerobically in MRS-glucose broth at $37^{\circ} \mathrm{C}$ overnight and unless otherwise stated, all further incubations were also performed under anaerobic conditions at $37^{\circ} \mathrm{C}$.

\section{Growth medium}

Modifications were made to the de Man-Rogosa-Sharpe (MRS) [56] medium by omitting the carbohydrate source (glucose) and meat extract. Carbohydrate-free MRS (cfMRS) was used as a basal growth medium to study the ability of Lactobacillus ruminis strains to utilise various carbohydrates, because it contains no additional carbohydrates and lacks Lab Lembco as a source of carbohydrates. The cfMRS medium contained the following components $\left(\mathrm{gL}^{-1}\right)$ : bacteriological peptone (Oxoid) 10.0, yeast extract (Fluka) 5.0, sodium acetate (Sigma) 5.0, ammonium citrate (Sigma) 2.0, potassium phosphate (Sigma) 2.0, magnesium sulphate (BDH Chemical) 0.2, Manganese sulphate (BDH Chemical) 0.05 . The medium also includes Tween 80 (Sigma) $1 \mathrm{ml} \mathrm{litre}^{-}$

${ }^{1}$. The $\mathrm{pH}$ was adjusted to between 6.2 and 6.5 and the medium was sterilised at $121^{\circ} \mathrm{C}$ for 15 minutes. Carbohydrate-free MRS was unable to support bacterial growth above an $\mathrm{OD}_{600 \mathrm{~nm}}$ of 0.1 for any of the strains tested.

\section{Carbohydrates and prebiotics}

Fifty- two carbohydrates were used in this study (Additional file 28). Stock solutions of the 50 carbohydrates were filter-sterilized $(0.45 \mu \mathrm{m})$ (Sarstedt) into the cfMRS basal medium to yield a concentration of $0.5 \%(\mathrm{v} / \mathrm{v})$ for use in the fermentation tests.

\section{Growth measurements}

The fermentation profiles of the various strains were determined using optical density (OD) measurements.
The sterile carbohydrate supplemented MRS media was added to the wells of 96 well microtiter plates. The medium in the wells was inoculated with $1 \%(\mathrm{v} / \mathrm{v})$ of the overnight bacterial culture in MRS-glucose. The OD values of the 96 well microtiter plate wells were read using a Synergy 2 plate reader (BioTek Instruments, Inc., Vermont, US). The inoculated microtiter plates were incubated anaerobically at $37^{\circ} \mathrm{C}$ and $\mathrm{OD}$ readings were taken before and after a 48 hour period [57]. The mean OD readings, standard deviations and standard errors were calculated using technical triplicate data from biological duplicate experiments.

\section{Lactobacillus ruminis genome sequencing and assembly}

The genome sequencing, assembly and detailed annotation of the L. ruminis ATCC 27782 and 25644 genomes will be described elsewhere in this volume (Forde et al, manuscript in preparation). In brief, a hybrid next-generation strategy generated 28 -fold coverage of the ATCC 27782 genome by 454 pyrosequencing, complimented by 217 -fold coverage with Illumina paired-end sequences. The assembly of $L$. ruminis ATCC 27782 is a finished genome; the genome assembly of L. ruminis ATCC 25644 a high-quality draft [58].

\section{Bioinformatic analysis and gene annotation}

The Artemis program [59] was used to visualise and identify carbohydrate metabolism genes in the genome of Lactobacillus ruminis ATCC 25644 and ATCC 27782 [60]. Open reading frames were predicted using Glimmer 3 [61]. Each carbohydrate utilisation enzyme, predicted from opening reading frames (ORF), was assigned a KEGG orthology (KO) identifier by KAAS and graphical representations for each metabolic pathway were generated [62]. The TMHMM 2.0 server was used to predict the transmembrane helices of proteins, which were identified from annotation as putative carbohydrate transporters. THHMM 2.0 uses Hidden Markov models to predict the proteins topology with a high degree of accuracy [63]. TransTermHP [64] was used to predict rhoindependent transcriptional terminators. Comparisons to other Lactobacillus genomes were made using data available from both NCBI [65] and KEGG Organisms [66].

Sequence data availability and accession numbers The finished genome of ATCC 27782 is available under accession number XXYYZZ123. The draft genome of ATCC 25644 is available under accession number CCGGHHIIUU.

\section{Additional material}

Additional file 1: Fermentation profiles for nine Lactobacillus ruminis strains 
Additional file 2: Growth profile for $L$. ruminis strain $L 5$

Additional file 3: Growth profile for $L$. ruminis strain $\$ 21$

Additional file 4: Growth profile for L. ruminis strain $\$ 23$

Additional file 5: Growth profile for L. ruminis strain $\mathrm{S36}$

Additional file 6: Growth profile for L. ruminis strain $\$ 38$

Additional file 7: Growth profile for L. ruminis strain ATCC 25644

Additional file 8: Growth profile for L. ruminis strain ATCC 27780T

Additional file 9: Growth profile for L. ruminis strain ATCC 27781

Additional file 10: Growth profile for $L$. ruminis strain ATCC 27782

Additional file 11: Glycolysis map representing enzymes present in L. ruminis ATCC 25644 or ATCC 27782.

Additional file 12: Citrate cycle map representing enzymes present in L. ruminis ATCC 25644 or ATCC 27782.

Additional file 13: Pentose phosphate pathway map representing enzymes present in L. ruminis ATCC 25644 or ATCC 27782.

Additional file 14: Pentose and glucuronate interconversions map representing enzymes present in L. ruminis ATCC 25644 or ATCC 27782.

Additional file 15: Fructose and Mannose metabolism map representing enzymes present in L. ruminis ATCC 25644 or ATCC 27782.

Additional file 16: Galactose metabolism map representing enzymes present in L. ruminis ATCC 25644 or ATCC 27782.

Additional file 17: Ascorbate and aldarate metabolism map representing enzymes present in L. ruminis ATCC 25644 or ATCC 27782.

Additional file 18: Starch and sucrose metabolism map representing enzymes present in L. ruminis ATCC 25644 or ATCC 27782.

Additional file 19: Amino and nucleotide sugar metabolism map representing enzymes present in L. ruminis ATCC 25644 or ATCC 27782.

Additional file 20: Inositol Phosphate metabolism map representing enzymes present in L. ruminis ATCC 25644 or ATCC 27782.

Additional file 21: Pyruvate metabolism map representing enzymes present in L. ruminis ATCC 25644 or ATCC 27782.

Additional file 22: Glyoxylate and Dicarboxylate metabolism map representing enzymes present in L. ruminis ATCC 25644 or ATCC 27782.

Additional file 23: Propanoate metabolic map representing enzymes present in L. ruminis ATCC 25644 or ATCC 27782.

Additional file 24: Butanoate metabolic map representing enzymes present in L. ruminis ATCC 25644 or ATCC 27782.

Additional file 25: $A B C$ transporters map representing enzymes present in L. ruminis ATCC 25644 or ATCC 27782.

Additional file 26: Phosphotransferase system map representing enzymes present in L. ruminis ATCC 25644 or ATCC 27782.

Additional file 27: Operons in the genome of $L$. ruminis ATCC 27782 associated with prebiotic utilisation

Additional file 28: Carbohydrates used in this study

\section{Acknowledgements}

This work was supported by a Principal Investigator Award (07/IN.1/B1780) from Science Foundation Ireland to P.W. OToole.

This article has been published as part of Microbial Cell Factories Volume 10 Supplement 1, 2011: Proceedings of the 10th Symposium on Lactic Acid Bacterium. The full contents of the supplement are available online at http:// www.microbialcellfactories.com/supplements/10/S1.
Author details

${ }^{1}$ Teagasc Food Research Centre, Moorepark, Fermoy, Co. Cork, Ireland.

2Department Microbiology, University College Cork, Ireland.

\section{Authors' contributions}

MMOD designed the experiments and drafted the manuscript. BMF carried out the genome sequencing and assembly of both $L$. ruminis genomes and provided the output of both the TMHMM 2.0 server and TransTerm HP. BAN initiated the genome sequencing and participated in assembly of the genomes. PWOT and RPR conceived the study, designed the research, and contributed to writing the manuscript.

\section{Competing interests}

The authors declare they have no competing interest.

Published: 30 August 2011

\section{References}

1. Qin J, Li R, Raes J, Arumugam M, Burgdorf KS, Manichanh C, Nielsen T, Pons $N$, Levenez F, Yamada $T$, et al: A human gut microbial gene catalogue established by metagenomic sequencing. Nature 2010 464:59-65.

2. OToole PW, Claesson MJ: Gut microbiota: Changes throughout the lifespan from infancy to elderly. International Dairy Journal 20:281-291.

3. Reuter G: The Lactobacillus and Bifidobacterium microflora of the human intestine: composition and succession. Curr Issues Intest Microbiol 2001 2:43-53.

4. Al Jassim RA: Lactobacillus ruminis is a predominant lactic acid producing bacterium in the caecum and rectum of the pig. Lett Appl Microbiol 2003, 37:213-217.

5. Sharpe ME, Latham MJ, Garvie El, Zirngibl J, Kandler O: Two new species of Lactobacillus isolated from the bovine rumen, Lactobacillus ruminis sp. nov. and Lactobacillus vitulinus sp.nov. J Gen Microbiol 1973, 77:37-49.

6. Krieg NR, Holt JG: Bergey's manual of systematic bacteriology. 1984

7. Taweechotipatr M, lyer C, Spinler JK, Versalovic J, Tumwasorn S: Lactobacillus saerimneri and Lactobacillus ruminis: novel human-derived probiotic strains with immunomodulatory activities. FEMS Microbiology Letters 2009, 293:65-72

8. Yin Q, Zheng Q: Isolation and identification of the dominant Lactobacillus in gut and faeces of pigs using carbohydrate fermentation and $16 \mathrm{~S}$ rDNA analysis. J Biosci Bioeng 2005, 99:68-71.

9. Report on Joint FAO/WHO expert consultation on evaluation of health and nutritional properties of probiotics in food Including powder milk with live lactic acid bacteria. [ftp://ftp.fao.org/es/esn/food/ probio_report_en.pdf].

10. Bezkorovainy A: Probiotics: determinants of survival and growth in the gut. Am J Clin Nutr 2001, 73:399S-405.

11. Gibson GR, Scott KP, Rastall RA, Tuohy KM, Hotchkiss A, DubertFerrandon A, Gareau M, Murphy EF, Saulnier D, Loh G: Dietary prebiotics: current status and new definition. Food Science \& Technology Bulletin: Functional Foods 2010, 7:1-19.

12. Gibson GR, Roberfroid MB: Dietary modulation of the human colonic microbiota: Introducing the concept of prebiotics. J Nutr 1995, 125:1401-1412

13. Swennen K, Courtin C, Delcour J: Non-digestible oligosaccharides with prebiotic properties. Critical Reviews in Food Science \& Nutrition 2006, 46:459-471.

14. Manning TS, Gibson GR: Microbial-gut interactions in health and disease. Prebiotics. Best Pract Res Clin Gastroenterol 2004, 18:287-298.

15. Gibson GR, Probert HM, Loo JV, Rastall RA, Roberfroid MB: Dietary modulation of the human colonic microbiota: updating the concept of prebiotics. Nutr Res Rev 2004, 17:259-275.

16. Henrissat B: A classification of glycosyl hydrolases based on amino acid sequence similarities. Biochem J 1991, 280:309-316.

17. Henrissat B, Bairoch A: New families in the classification of glycosyl hydrolases based on amino acid sequence similarities. Biochem J 1993, 293(Pt 3):781-788

18. Henrissat $B$, Bairoch $A$ : Updating the sequence-based classification of glycosyl hydrolases. Biochem J 1996, 316(Pt 2):695-696. 
19. Saulnier DM, Molenaar D, de Vos WM, Gibson GR, Kolida S: Identification of prebiotic fructooligosaccharide metabolism in Lactobacillus plantarum WCFS1 through microarrays. Appl Environ Microbiol 2007, 73:1753-1765.

20. Barrangou R, Altermann E, Hutkins R, Cano R, Klaenhammer TR: Functional and comparative genomic analyses of an operon involved in fructooligosaccharide utilization by Lactobacillus acidophilus. Proc Nat Acad Sci U S A 2003, 100:8957-8962.

21. Goh YJ, Zhang C, Benson AK, Schlegel V, Lee J-H, Hutkins RW: Identification of a putative operon involved in fructooligosaccharide utilization by Lactobacillus paracasei. Appl Environ Microbiol 2006, 72:7518-7530

22. Stentz R, Loizel C, Malleret C, Zagorec M: Development of genetic tools for Lactobacillus sakei: Disruption of the beta-galactosidase gene and use of lacZ as a reporter gene to study regulation of the putative copper ATPase, AtkB. Appl Environ Microbiol 2000, 66:4272-4278.

23. Schmidt BF, Adams RM, Requadt C, Power S, Mainzer SE: Expression and nucleotide sequence of the Lactobacillus bulgaricus beta-galactosidase gene cloned in Escherichia coli. J Bacteriol 1989, 171:625-635.

24. Corral J, Bañuelos O, Adrio J, Velasco J: Cloning and characterization of a $\beta$-galactosidase encoding region in Lactobacillus coryniformis CECT 5711. Applied microbiology and biotechnology 2006, 73:640-646.

25. Nguyen T-h, Splechtna B, Steinböck M, Kneifel W, Lettner HP, Kulbe KD, Haltrich D: Purification and characterization of two novel betagalactosidases from Lactobacillus reuteri. Journal of Agricultural and Food Chemistry 2006, 54:4989-4998.

26. Spano G, Rinaldi A, Ugliano M, Moio L, Beneduce L, Massa S: A betaglucosidase gene isolated from wine Lactobacillus plantarum is regulated by abiotic stresses. Journal of Applied Microbiology 2005, 98:855-861.

27. Silvestroni A, Connes C, Sesma F, de Giori GS, Piard J-C: Characterization of the melA locus for a-galactosidase in Lactobacillus plantarum. Appl Environ Microbiol 2002, 68:5464-5471.

28. Tzortzis G, Jay AJ, Baillon MLA, Gibson GR, Rastall RA: Synthesis of agalactooligosaccharides with a-galactosidase from Lactobacillus reuteri of canine origin. Applied microbiology and biotechnology 2003, 63:286-292.

29. De Cort S, Kumara HMCS, Verachtert H: Localization and characterization of alpha-glucosidase activity in Lactobacillus brevis. Appl Environ Microbiol 1994, 60:3074-3078.

30. Li KB, Chan KY: Production and properties of alpha-glucosidase from Lactobacillus acidophilus. Appl Environ Microbiol 1983, 46:1380-1387.

31. Chaillou S, Lokman BC, Leer RJ, Posthuma C, Postma PW, Pouwels PH: Cloning, sequence analysis, and characterization of the genes involved in isoprimeverose metabolism in Lactobacillus pentosus. J Bacteriol 1998, 180:2312-2320

32. Human Microbiome Project. [http://nihroadmap.nih.gov/hmp/]

33. Snel B, Bork P, Huynen MA: The identification of functional modules from the genomic association of genes. Proceedings of the National Academy of Sciences of the United States of America 2002, 99:5890-5895

34. Saier MH Jr.: A functional-phylogenetic classification system for transmembrane solute transporters. Microbiol Mol Biol Rev 2000, 64:354-411.

35. Antonio MA, Hawes SE, Hillier SL: The identification of vaginal Lactobacillus species and the demographic and microbiologic characteristics of women colonized by these species. J Infect Dis 1999, 180:1950-1956

36. Delgado S, Suarez A, Otero L, Mayo B: Variation of microbiological and biochemical parameters in the faeces of two healthy people over a 15 day period. Eur J Nutr 2004, 43:375-380

37. Kimura K, Nishio T, Mizoguchi C, Koizumi A: Analysis of the composition of lactobacilli in humans. Bioscience and Microflora 2010, 29:47-50

38. Kovalenko NK, Golovach TN, Kvasnikov El: Lactic bacteria in the digestive tract of poultry. Mikrobiologiia 1989, 58:137-143.

39. Mueller RE, lannotti EL, Asplund JM: Isolation and identification of adherent epimural bacteria during succession in young lambs. Appl Environ Microbiol 1984, 47:724-730.

40. Mathiesen SD, Orpin CG, Greenwood Y, Blix AS: Seasonal changes in the cecal microflora of the high-arctic Svalbard reindeer (Rangifer tarandus platyrhynchus). Appl Environ Microbiol 1987, 53:114-118.

41. Hidetoshi M, Akiyo N, Mitsuharu S, Hidehiro TOH, Fumihiko N, Masahiro N, Shin H, Yukio K, Misako T, Hideto T, et al: Lactobacillus hayakitensis, L. equigenerosi and $L$. equi, predominant lactobacilli in the intestinal flora of healthy thoroughbreds. Animal Science Journal 2009, 80:339-346.
42. Vörös A: Diet related changes in the gastrointestinal microbiota of horses. Masters Swedish University of Agricultural Sciences, Dept of Animal Nutrition and Management; 2008

43. Willing B, Voros A, Roos S, Jones C, Jansson A, Lindberg JE: Changes in faecal bacteria associated with concentrate and forage-only diets fed to horses in training. Equine Vet J 2009, 41:908-914.

44. Desai AR, Musil KM, Carr AP, Hill JE: Characterization and quantification of feline fecal microbiota using cpn60 sequence-based methods and investigation of animal-to-animal variation in microbial population structure. Veterinary Microbiology 2009, 137:120-128.

45. Ritchie LE, Burke KF, Garcia-Mazcorro JF, Steiner JM, Suchodolski JS: Characterization of fecal microbiota in cats using universal 16S rRNA gene and group-specific primers for Lactobacillus and Bifidobacterium spp. Veterinary Microbiology 2009, Corrected Proof.

46. Greetham HL, Giffard C, Hutson RA, Collins MD, Gibson GR: Bacteriology of the Labrador dog gut: a cultural and genotypic approach. Journal of Applied Microbiology 2002, 93:640-646.

47. Xenoulis PG, Gray PL, Brightsmith D, Palculict B, Hoppes S, Steiner JM, Tizard I, Suchodolski JS: Molecular characterization of the cloacal microbiota of wild and captive parrots. Veterinary Microbiology 2010, 146:320-325

48. Kandler O, Weiss N: Genus Lactobacillus. Bergey's manual of systematic bacteriology 1986, 2:1209-1234.

49. Tanasupawat S, Shida O, Okada S, Komagata K: Lactobacillus acidipiscis sp. nov. and Weissella thailandensis sp. nov., isolated from fermented fish in Thailand. Int I Syst Evol Microbiol 2000, 50:1479-1485.

50. Kato Y, Sakala RM, Hayashidani H, Kiuchi A, Kaneuchi C, Ogawa M: Lactobacillus algidus sp. nov., a psychrophilic lactic acid bacterium isolated from vacuum-packaged refrigerated beef. Int J Syst Evol Microbiol 2000, 50:1143-1149.

51. Lina BAR, Jonker D, Kozianowski G: Isomaltulose (Palatinose ${ }^{\oplus}$ ): a review of biological and toxicological studies. Food and Chemical Toxicology 2002, 40:1375-1381.

52. Saarela M, Hallamaa K, Mattila-Sandholm T, Mättö J: The effect of lactose derivatives lactulose, lactitol and lactobionic acid on the functional and technological properties of potentially probiotic Lactobacillus strains. International Dairy Journal 2003, 13:291-302.

53. Snart J, Bibiloni R, Grayson T, Lay C, Zhang H, Allison GE, Laverdiere JK, Temelli F, Vasanthan T, Bell R, Tannock GW: Supplementation of the diet with high-viscosity beta-glucan results in enrichment for lactobacilli in the rat cecum. Appl Environ Microbiol 2006, 72:1925-1931.

54. Kneifel W, Rajal A, Kulbe KD: In vitro growth behaviour of probiotic bacteria in culture media with carbohydrates of prebiotic importance. Microbial Ecology in Health \& Disease 2000, 12:27-34.

55. Makras L, Van Acker G, De Vuyst L: Lactobacillus paracasei subsp. paracasei 8700:2 degrades inulin-type fructans exhibiting different degrees of polymerization. Appl Environ Microbiol 2005, 71:6531-6537.

56. De Man JC, Rogosa M, Sharpe ME: A medium for the cultivation of lactobacilli. Journal of Applied Microbiology 1960, 23:130-135.

57. Brewster JD: A simple micro-growth assay for enumerating bacteria. Journal of Microbiological Methods 2003, 53:77-86.

58. Chain PSG, Grafham DV, Fulton RS, FitzGerald MG, Hostetler J, Muzny D, Ali J, Birren B, Bruce DC, Buhay C, et al: Genome Project Standards in a New Era of Sequencing. Science 2009, 326:236-237.

59. Rutherford K, Parkhill J, Crook J, Horsnell T, Rice P, Rajandream M-Al, Barrell B: Artemis: sequence visualization and annotation. Bioinformatics 2000, 16:944-945.

60. Mural RJ: ARTEMIS: a tool for displaying and annotating DNA sequence. Brief Bioinform 2000, 1:199-200.

61. Delcher AL, Bratke KA, Powers EC, Salzberg SL: Identifying bacterial genes and endosymbiont DNA with Glimmer. Bioinformatics 2007, 23:673-679.

62. Moriya Y, Itoh M, Okuda S, Yoshizawa AC, Kanehisa M: KAAS: an automatic genome annotation and pathway reconstruction server. Nucleic Acids Res 2007, 35:W182-185

63. Krogh A, Larsson B, von Heijne G, Sonnhammer ELL: Predicting transmembrane protein topology with a hidden markov model: application to complete genomes. Journal of Molecular Biology 2001, 305:567-580.

64. Kingsford C, Ayanbule K, Salzberg S: Rapid, accurate, computational discovery of Rho-independent transcription terminators illuminates their relationship to DNA uptake. Genome Biology 2007, 8:R22. 
65. NCBI Genome Homepage. [http://www.ncbi.nlm.nih.gov/sites/genome].

66. KEGG Organisms: Complete Genomes. [http://kegg.jp/kegg/catalog/ org_list.html].

doi:10.1186/1475-2859-10-S1-S12

Cite this article as: $\mathrm{O}^{\prime}$ Donnell et al: Carbohydrate catabolic flexibility in the mammalian intestinal commensal Lactobacillus ruminis revealed by fermentation studies aligned to genome annotations. Microbial Cell Factories 2011 10(Suppl 1):S12.

Submit your next manuscript to BioMed Central and take full advantage of:

- Convenient online submission

- Thorough peer review

- No space constraints or color figure charges

- Immediate publication on acceptance

- Inclusion in PubMed, CAS, Scopus and Google Scholar

- Research which is freely available for redistribution

Submit your manuscript at www.biomedcentral.com/submit
() Biomed Central 